\title{
BAYESIAN AND NON-BAYESIAN ESTIMATION FROM WEIBULL-UNIFORM DISTRIBUTION BASED ON UPPER RECORD VALUES
}

\author{
A. AMIN ESSAM ${ }^{1,2}$ and A. ABDEL-AZIZ ALAA ${ }^{3}$ \\ ${ }^{1}$ Department of Mathematics and Statistics \\ College of Science \\ Al-Imam Mohammad Ibn Saud Islamic University \\ Saudi Arabia \\ e-mail: ess_amin@yahoo.com \\ 2Department of Mathematical Statistics \\ Institute of Statistical Studies and Research \\ Cairo University \\ Egypt \\ 3Department of Applied Statistics and Econometrics \\ Institute of Statistical Studies and Research \\ Cairo University \\ Egypt \\ e-mail: alaa_mnn@yahoo.com
}




\begin{abstract}
This paper develops a Bayesian and non-Bayesian analysis in the context of upper record values from the Weibull-Uniform (WU) distribution. The maximum likelihood estimates and Bayes estimates based on upper record values are derived for the unknown parameters. The Bayes estimates are obtained with respect to both squared error loss and zero one loss functions. To compare the different estimates, the relative absolute error and mean squared error are calculated through simulation study.
\end{abstract}

\title{
1. Introduction
}

Record values and associated statistics are of great importance in several real-life applications involving weather, economic, and sports data "Olympic records or world records in sport". Also in industry, many products fail under stress, for example, a wooden beam breaks when sufficient perpendicular force is applied to it, an electronic component ceases to function in an environment of too high temperature, and a battery dies under the stress of time. But the precise breaking stress or failure point varies even among identical items. Hence, in such experiments, measurements may be made sequentially and only the record values are observed. Thus, the number of measurements made is considerably smaller than the complete sample size. This "measurement saving" can be important when the measurements of these experiments are costly if the entire sample was destroyed. For more examples, see Gulati and Padgett [1]. There are also situations in which an observation is stored only if it is a record value. These include studies in meteorology, hydrology, seismology, athletic events, and mining. In recent years, there has been much work on parametric and nonparametric inference based on record values. Among others are Resnick [2], Nagaraja [3], Ahsanullah [4, 5], Arnold et al. [6, 7], Gulati and Padgett [1], Raqab and Ahsanullah [8], and Soliman et al. [9].

The statistical study of record values started with Chandler [10], he formulated the theory of record values as a model for successive extremes in a sequence of independently and identically random variables. Feller [11] gave some examples of record values with respect to gambling 
problems. Resnick [12] discussed the asymptotic theory of records. Theory of record values and its distributional properties have been extensively studied in the literature, for example, see Ahsanullah [5], Nagaraja [3], and Balakrishnan and Ahsanullah [13].

The Weibull-uniform (WU) distribution is defined in the following way (Bourguignon et al. [14]). It has the distribution function given by

$$
F(x)=1-e^{-\alpha\left(\frac{x}{\lambda-x}\right)^{\beta}}, \quad 0<x<\lambda,
$$

and therefore its probability density function (pdf) of the form

$$
f(x)=\frac{\alpha \beta \lambda}{(\lambda-x)^{2}}\left(\frac{x}{\lambda-x}\right)^{\beta-1} e^{-\alpha\left(\frac{x}{\lambda-x}\right)^{\beta}}, \quad 0<x<\lambda .
$$

The corresponding survival function is

$$
\bar{F}(x)=S(x)=1-F(x)=e^{-\alpha\left(\frac{x}{\lambda-x}\right)^{\beta}}, \quad 0<x<\lambda,
$$

and the hazard function is

$$
h(x)=\frac{\alpha \beta \lambda}{(\lambda-x)^{2}}\left(\frac{x}{\lambda-x}\right)^{\beta-1},
$$

where $\alpha, \beta, \lambda>0$. Here $\alpha$ denotes the scale parameter, $\beta$ denotes the shape parameter, and $\lambda$ is the location parameter.

Let $X_{1}, X_{2}, \ldots$ be a sequence of independent and identically distributed (i.i.d) random variables with cumulative function (cdf) $F(x)$ and probability density function (pdf) $f(x)$. Set $Y_{n}=\max \left\{X_{1}, X_{2}, \cdots, X_{n}\right\}$ for $n \geq 1$. We say $X_{j}$ is an upper record value of this sequence, if $Y_{j+1}>Y_{j}$. By definition, $X_{1}$ is an upper record values. The indices at which the upper record values occur are called upper record times $\{U(m), m \geq 0\}$, where $U(0)=1$ and $U(m)=\min \{j: j>U(m-1)$, $\left.X_{j}>X_{U(m-1)}\right\}$. Then $R_{m}=X_{U(m)}, m \geq 0$ are called the upper record values (see Ahsanullah [5]). 
Let $R_{0}, R_{1}, R_{2}, \ldots, R_{m}$ represent the first $(m+1)$ upper record values arising from a sequence $\left\{X_{i}\right\}$ of iid Weibull-uniform variable. Then the pdf of $R_{m}$ is given by

$$
f_{R_{m}}\left(r_{m}\right)=\frac{1}{\Gamma(m+1)}\left[-\ln \bar{F}\left(r_{m}\right)\right]^{m} f\left(r_{m}\right), \quad 0<r_{m}<\lambda, \quad m=0,1,2, \ldots
$$

while the likelihood function based on the $(m+1)$ upper record values $R_{0}, R_{1}, R_{2}, \ldots, R_{m}$ is given by

$$
f\left(r_{0}, r_{1}, \ldots, r_{m}\right)=\prod_{i=0}^{m-1} \frac{f\left(r_{i}\right)}{\bar{F}\left(r_{i}\right)} f\left(r_{m}\right),
$$

where $0<r_{0}<r_{1}<\ldots<r_{m-1}<r_{m}<\lambda$.

This paper, is devoted to obtain and compare several techniques of estimation based on upper record values. Section 2 contains the maximum likelihood estimators for the two unknown parameters of the Weibull-uniform distribution based on upper record values, also, Fisher information matrix will be obtained. In Section 3, Bayes estimators of the parameters are derived. The Bayes estimates are obtained by using both the zero one loss function and the squared error loss function. In Section 4, numerical study for the theoretical results will be provided. Finally, some remarks and a brief summary of the results will be concluded in Section 5.

\section{Maximum Likelihood Estimation}

Let $X_{1}, X_{2}, \ldots$ be an infinite sequence of independent and identically distributed random variables having the Weibull-uniform (WU) distribution (1.1). Let $R_{0}, R_{1}, R_{2}, \ldots, R_{m}$ be the first $(m+1)$ upper record values arising from a sequence $\left\{X_{i}\right\}$ of iid Weibull-uniform variables. Substituting from (1.1) to (1.3) in (1.6), then the likelihood function is given by 


$$
\begin{gathered}
L(\Theta)=(\beta \lambda \alpha)^{m+1} \prod_{i=0}^{m}\left(r_{i}^{\beta-1}\left(\frac{1}{\lambda-r_{m}}\right)^{\beta+1}\right) e^{-\alpha\left(\frac{r_{m}}{\lambda-r_{m}}\right)^{\beta},} \\
0<r_{0}<r_{1}<\cdots<r_{m}<\lambda,
\end{gathered}
$$

where $\Theta=(\alpha, \beta)$ and $\lambda$ is known location parameter. Taking the logarithm of the likelihood function (2.1), then

$$
\begin{aligned}
\operatorname{LnL}(\Theta)= & (m+1) \ln (\alpha \beta \lambda)+(\beta-1) \sum_{i=0}^{m} \ln \left(r_{i}\right)-(\beta+1) \sum_{i=0}^{m} \ln \left(\lambda-r_{i}\right) \\
& -\alpha\left(\frac{r_{m}}{\lambda-r_{m}}\right)^{\beta} .
\end{aligned}
$$

Differentiate (2.2) with respect to $\alpha$ and $\beta$, then

$$
\frac{\partial \operatorname{LnL}(\Theta)}{\partial \alpha}=\frac{(m+1)}{\alpha}-\left(\frac{r_{m}}{\lambda-r_{m}}\right)^{\beta}
$$

and

$$
\frac{\partial \operatorname{Ln} L(\Theta)}{\partial \beta}=\frac{(m+1)}{\beta}+\sum_{i=0}^{m} \ln \left(r_{i}\right)-\sum_{i=0}^{m} \ln \left(\lambda-r_{i}\right)-\alpha\left(\frac{r_{m}}{\lambda-r_{m}}\right)^{\beta} \ln \left(\frac{r_{m}}{\lambda-r_{m}}\right)
$$

Equating (2.3) and (2.4) by zero and solving with respect to $\alpha$ and $\beta$, then the maximum likelihood estimators $\hat{\alpha}$ and $\hat{\beta}$ for $\alpha$ and $\beta$ will be

$$
\hat{\alpha}=(m+1)\left(\frac{\lambda-r_{m}}{r_{m}}\right)^{\hat{\beta}}
$$

and

$$
\hat{\beta}=\frac{(m+1)}{(m+1) \ln \left(\frac{r_{m}}{\lambda-r_{m}}\right)-\sum_{i=0}^{m} \ln \left(\lambda-r_{i}\right)+\sum_{i=0}^{m} \ln \left(r_{i}\right)} .
$$


One can solve (2.6) to obtain $\hat{\beta}$ and then back substitution in (2.5) to find

$\hat{\alpha}$. The second derivatives of the log-likelihood function (2.1) are given by

$$
\begin{gathered}
\frac{\partial^{2} \operatorname{Ln}(L(\Theta))}{\partial \alpha^{2}}=-\frac{(m+1)}{\alpha^{2}}, \\
\frac{\partial^{2} \operatorname{Ln}(L(\Theta))}{\partial \beta^{2}}=\frac{-(m+1)}{\beta^{2}}-\alpha\left(\frac{r_{m}}{\lambda-r_{m}}\right)^{\beta}\left[\ln \left(\frac{r_{m}}{\lambda-r_{m}}\right)\right]^{2},
\end{gathered}
$$

and

$$
\frac{\partial^{2} \operatorname{Ln}(L(\Theta))}{\partial \alpha \partial \beta}=-\left(\frac{r_{m}}{\lambda-r_{m}}\right)^{\beta} \ln \left(\frac{r_{m}}{\lambda-r_{m}}\right)
$$

Hence, the observed information matrix defined by Lawless [15] as the second derivatives of the log-likelihood function evaluated at the maximum likelihood estimates of the parameters, therefore,

$$
\hat{I}(\hat{\alpha}, \hat{\beta})=\left[\begin{array}{cc}
-\frac{\partial^{2} \ln L}{\partial \alpha^{2}} & -\frac{\partial^{2} \ln L}{\partial \alpha \partial \beta} \\
-\frac{\partial^{2} \ln L}{\partial \beta \partial \alpha} & -\frac{\partial^{2} \ln L}{\partial \beta^{2}}
\end{array}\right]_{\alpha=\hat{\alpha}, \beta=\hat{\beta}} .
$$

Consequently, the inverse of the observed Fisher information matrix will be

$$
\hat{I}^{-1}(\hat{\alpha}, \hat{\beta})=\left[\begin{array}{cc}
\operatorname{Var}(\hat{\alpha}) & \operatorname{Cov}(\hat{\alpha}, \hat{\beta}) \\
\operatorname{Cov}(\hat{\beta}, \hat{\alpha}) & \operatorname{Var}(\hat{\beta})
\end{array}\right] .
$$

For large sample, the matrix $\hat{I}^{-1}(\hat{\alpha}, \hat{\beta})$ is an approximated variancecovariance matrix of the maximum likelihood estimators $\hat{\alpha}$ and $\hat{\beta}$.

\section{Special case}

When the shape parameter $\beta$ is known, then

$$
\hat{\alpha}_{1}=(m+1)\left(\frac{\lambda-r_{m}}{r_{m}}\right)^{\beta} \text {. }
$$


Also, the second derivatives of the log-likelihood function (2.2) is given by

$$
\frac{\partial^{2} \operatorname{Ln}(L(\Theta))}{\partial \alpha^{2}}=-\frac{(m+1)}{\alpha^{2}}
$$

For large sample, the matrix $\hat{I}^{-1}\left(\hat{\alpha}_{1}\right)$ is an approximated variance of the maximum likelihood estimator $\hat{\alpha}_{1}$, where

$$
\hat{I}^{-1}\left(\hat{\alpha}_{1}\right)=\left[-\frac{1}{\frac{\partial^{2} \ln L}{\partial \alpha^{2}}}\right]_{\alpha=\hat{\alpha}_{1}} \text {. }
$$

\section{Bayesian Estimation Based on Record Values}

In recent decades, the Bayesian viewpoint has received frequent attention for analyzing failure data and other time-to-event data, and has been often proposed as a valid alternative to traditional statistical perspectives. Bayesian methods usually require less sample data to achieve the same quality of inferences than methods based on sampling theory. In this section, Bayesian estimation for the parameter $\alpha$ and $\beta$, based on upper record values will be discussed. Here, two cases are considered: First, the case when shape parameter is known and second, the case when two parameters are both unknown. The Bayes estimators are studied under squared error and zero one loss functions.

\subsection{Known shape parameter}

Under the assumption that the shape parameter $\beta$ is known, assume a gamma $G(a, b)$ conjugate prior for $\alpha$ as

$$
\pi(\alpha) \propto \alpha^{a-1} e^{-b \alpha}, \quad \alpha>0, \quad a, b>0 .
$$

Combining the likelihood function (2.1) and the prior density (3.1), the posterior density function of $\alpha$ is given by

$$
\pi^{*}(\alpha) \propto \alpha^{m+a} \prod_{i=0}^{m}\left(r_{i}^{\beta-1}\left(\frac{1}{\lambda-r_{m}}\right)^{\beta+1}\right) e^{-\alpha\left(b+\left(\frac{r_{m}}{\lambda-r_{m}}\right)^{\beta}\right)} .
$$




\subsubsection{Bayes estimator based on zero one loss function}

Taking the logarithm of (3.2), then

$$
\operatorname{Ln} \pi^{*}(\alpha) \propto(m+a) \ln (\alpha)-\alpha\left(b+\left(\frac{r_{m}}{\lambda-r_{m}}\right)^{\beta}\right) .
$$

Differentiate (3.3) with respect to $\alpha$ and equating by zero then solve for $\alpha$, one can obtain the Bayes posterior mode $\hat{\alpha}_{2}$ for $\alpha$ as

$$
\hat{\alpha}_{2}=(m+a)\left(\left(b+\left(\frac{r_{m}}{\lambda-r_{m}}\right)^{\beta}\right)\right)^{-1} \text {. }
$$

\subsubsection{Bayes estimator based on squared error loss function}

From Equation (3.2), one can show that the posterior density function of $\alpha$ is the gamma function Gamma $\left(m+a+1,\left(b+\left(\frac{r_{m}}{\lambda-r_{m}}\right)^{\beta}\right)\right)$. Under a squared error loss function, the Bayes estimator $\hat{\alpha}_{3}$ is the posterior mean. Hence, the Bayes estimator $\hat{\alpha}_{3}$ of $\alpha$ is given by

$$
\hat{\alpha}_{3}=E(\alpha)=\frac{m+a+1}{\left(b+\left(\frac{r_{m}}{\lambda-r_{m}}\right)^{\beta}\right)}
$$

\subsection{Unknown scale and shape parameters}

It is well-known that, for the Bayes estimators, the performance depends on the form of the prior distribution and the loss function assumed. Under the assumption of the parameters $\alpha$ and $\beta$ are unknown, no analogous reduction via sufficiency is possible for the likelihood corresponding to a sample of records from the Weibull-uniform distribution. Also, specifying a general joint prior for $\alpha$ and $\beta$ leads to computational complexities. In trying to solve this problem, the prior density suggested by Ashour and Amin [16] will be used. The suggested 
prior for $\alpha$ is gamma $(a, b)$ distribution (3.1) and the suggested prior for $\beta$ is the Jaffery's prior on the interval $(0, \infty)$ also assume that the two parameters are independent, then the prior density for $\alpha$ and $\beta$ is given by

$$
\pi(\alpha, \beta) \propto \frac{\alpha^{a-1} e^{-a b}}{\beta^{1 / 2}}, \quad \alpha, \beta>0 .
$$

Combining the likelihood function (2.1) and (3.6), then the posterior distribution will be

$$
\pi^{*}(\alpha, \beta) \propto \beta^{m+\frac{1}{2}} \alpha^{m+a} \prod_{i=0}^{m}\left(r_{i}^{\beta-1}\left(\frac{1}{\lambda-r_{m}}\right)^{\beta+1}\right) e^{-\left(b+\left(\frac{r_{m}}{\lambda-r_{m}}\right)^{\beta}\right)} .
$$

\subsubsection{Bayes estimator based on zero one loss function}

Taking the logarithm of (3.7) to get

$$
\begin{aligned}
\operatorname{Ln} \pi^{*}(\alpha, \beta)= & \left(m+\frac{1}{2}\right) \ln (\beta)+(m+a) \ln (\alpha)+(\beta-1) \sum_{i=0}^{m} \ln \left(r_{i}\right) \\
& -(\beta+1) \sum_{i=0}^{m} \ln \left(\lambda-r_{i}\right)-\alpha\left(b+\left(\frac{r_{m}}{\lambda-r_{m}}\right)^{\beta}\right) .
\end{aligned}
$$

Differentiate (3.8) with respect to $\alpha$ and $\beta$, one can get the following:

$$
\frac{\partial \operatorname{Ln} \pi^{*}(\alpha, \beta)}{\partial \alpha}=\frac{(m+a)}{\alpha}-\left(b+\left(\frac{r_{m}}{\lambda-r_{m}}\right)^{\beta}\right),
$$

and

$$
\frac{\partial \operatorname{Ln} \pi^{*}(\alpha, \beta)}{\partial \beta}=\frac{\left(m+\frac{1}{2}\right)}{\beta}+\sum_{i=0}^{m} \ln \left(r_{i}\right)-\sum_{i=0}^{m} \ln \left(\lambda-r_{i}\right)-\alpha\left(\frac{r_{m}}{\lambda-r_{m}}\right)^{\beta} \ln \left(\frac{r_{m}}{\lambda-r_{m}}\right) .
$$


Equating (3.9) by zero and solving with respect to $\alpha$, then the Bayes posterior estimator $\hat{\alpha}_{4}$ for $\alpha$ will be

$$
\hat{\alpha}_{4}=(m+a)\left(\left(b+\left(\frac{r_{m}}{\lambda-r_{m}}\right)^{\hat{\beta}_{2}}\right)\right)^{-1}
$$

Substituting $\hat{\alpha}_{4}$ in Equation (3.10) and equating by zero, one can get

$$
\begin{aligned}
& \frac{\left(m+\frac{1}{2}\right)}{\hat{\beta}_{1}}+\sum_{i=0}^{m} \ln \left(r_{i}\right)-\sum_{i=0}^{m} \ln \left(\lambda-r_{i}\right) \\
& \quad-(m+a)\left(\left(b+\left(\frac{r_{m}}{\lambda-r_{m}}\right)^{\hat{\beta}_{1}}\right)\right)^{-1}\left(\frac{r_{m}}{\lambda-r_{m}}\right)^{\hat{\beta}_{1}} \ln \left(\frac{r_{m}}{\lambda-r_{m}}\right)=0 .
\end{aligned}
$$

Numerical technique is needed to solve Equation (3.12) to obtain $\hat{\beta}_{1}$.

\subsubsection{Marginal posterior for scale parameter}

Integrate (3.7) with respect to $\beta$ to get

$$
\pi^{*}(\alpha)=k_{1} \int_{0}^{\infty} \beta^{m+\frac{1}{2}} \alpha^{m+a} \prod_{i=0}^{m}\left(r_{i}^{\beta-1}\left(\frac{1}{\lambda-r_{m}}\right)^{\beta+1}\right) e^{-\left(b+\left(\frac{r_{m}}{\lambda-r_{m}}\right)^{\beta}\right)} d \beta
$$

where

$$
k_{1}=\frac{1}{\int_{0}^{\infty} \int_{0}^{\infty} \pi^{*}(\alpha, \beta) d \alpha d \beta}
$$

The posterior mean for $\alpha$ is

$$
\hat{\alpha}_{5}=E(\alpha)=k_{1} \int_{0}^{\infty} \int_{0}^{\infty} \beta^{m+\frac{1}{2}} \alpha^{m+a+1} \prod_{i=0}^{m}\left(r_{i}^{\beta-1}\left(\frac{1}{\lambda-r_{m}}\right)^{\beta+1}\right) e^{-\left(b+\left(\frac{r_{m}}{\lambda-r_{m}}\right)^{\beta}\right)} d \beta d \alpha .
$$

By solving (3.14) numerically, the Bayes estimator $\hat{\alpha}_{5}$ for $\alpha$ will be obtained. 


\subsubsection{Marginal posterior for shape parameter}

Integrate (3.7) with respect to $\alpha$, then

$$
\pi^{*}(\beta)=k_{1} \int_{0}^{\infty} \beta^{m+\frac{1}{2}} \alpha^{m+a} \prod_{i=0}^{m}\left(r_{i}^{\beta-1}\left(\frac{1}{\lambda-r_{m}}\right)^{\beta+1}\right) e^{-\left(b+\left(\frac{r_{m}}{\lambda-r_{m}}\right)^{\beta}\right)} d \alpha
$$

Similarly, the Bayes posterior estimator for $\beta$ is given by

$$
\hat{\beta}_{2}=E(\beta)=k_{1} \int_{0}^{\infty} \int_{0}^{\infty} \beta^{m+\frac{3}{2}} \alpha^{m+a} \prod_{i=0}^{m}\left(r_{i}^{\beta-1}\left(\frac{1}{\lambda-r_{m}}\right)^{\beta+1}\right) e^{-\left(b+\left(\frac{r_{m}}{\lambda-r_{m}}\right)^{\beta}\right)} d \alpha d \beta .
$$

By solving (3.16) numerically, one can obtain the Bayes estimator $\hat{\beta}_{2}$ for $\beta$.

\section{Simulation Study}

In order to compare the proposed Bayes estimators with the maximum likelihood estimators, a simulation study using different sample sizes and different values of the distribution parameters will be performed. All computations will be performed by using MathCAD(2001) program.

In the case when the two-parameter are unknown, a simulation study was conducted in order to compare the maximum likelihood estimates and Bayes estimates according to the following steps:

(1) For given values of the distribution parameters, generate $(m+1)$, $(m=3,5$, and 7) upper record values from the Weibull-uniform distribution (1.2).

(2) By solving Equations (2.5) and (2.6), one can obtain the maximum likelihood estimators for the unknown parameters $\alpha$ and $\beta$. 
(3) For the given values of the prior parameters, suggested by Congdon [17], $a=b=0.001$ and by using the Equation (3.11) to the Equation (3.16), the Bayes estimators for the unknown parameters will be obtained.

(4) Steps 1-3 are repeated 5,000 times, and the average relative absolute error and average mean square error for each method are calculated.

(5) Tables 1-3 contains the results when the two parameters are unknown which show

(i) the values of the distribution parameters;

(ii) the value of the average relative absolute error and average mean square error for the MLE and Bayes estimators. 
BAYESIAN AND NON-BAYESIAN ESTIMATION ...

Table 1.

\begin{tabular}{|c|c|c|c|c|c|c|}
\hline \multicolumn{7}{|c|}{ Relative absolute error and mean square error for $\hat{\alpha}$ and $\hat{\beta}$} \\
\hline$m$ & $\hat{\alpha}$ & $\hat{\alpha}_{4}$ & $\hat{\alpha}_{5}$ & $\hat{\beta}$ & $\hat{\beta}_{2}$ & $\hat{\beta}_{3}$ \\
\hline \multicolumn{7}{|c|}{ Simulated records with $(\alpha=0.5$ and $\beta=0.5)$} \\
\hline \multirow[t]{2}{*}{3} & 0.057 & 0.041 & 0.231 & 0.543 & 0.256 & 0.663 \\
\hline & $(0.195)$ & $(0.142)$ & $(0.303)$ & $(0.221)$ & $(0.078)$ & $(0.264)$ \\
\hline \multirow[t]{2}{*}{5} & 0.332 & 0.122 & 0.62 & 0.268 & 0.208 & 0.357 \\
\hline & $(0.262)$ & $(0.154)$ & $(0.375)$ & $(0.095)$ & $(0.052)$ & $(0.122)$ \\
\hline \multirow[t]{2}{*}{7} & 0.682 & 0.126 & 1.125 & 0.146 & 0.318 & 0.174 \\
\hline & $(0.336)$ & $(0.194)$ & $(0.64)$ & $(0.003)$ & $(0.068)$ & $(0.051)$ \\
\hline \multicolumn{7}{|c|}{ Simulated records with $(\alpha=1$ and $\beta=0.5)$} \\
\hline \multirow[t]{2}{*}{3} & 0.204 & 0.233 & 0.241 & 0.608 & 0.053 & 0.664 \\
\hline & $(0.313)$ & $(0.31)$ & $(0.354)$ & $(0.739)$ & $(0.192)$ & $(0.264)$ \\
\hline \multirow[t]{2}{*}{5} & 0.045 & 0.146 & 0.457 & 0.373 & 0.475 & 0.358 \\
\hline & $(0.261)$ & $(0.306)$ & $(0.838)$ & $(0.111)$ & $(0.157)$ & $(0.122)$ \\
\hline \multirow[t]{2}{*}{7} & 0.237 & 0.024 & 0.906 & 0.285 & 0.372 & 0.147 \\
\hline & $(0.272)$ & $(0.241)$ & $(1.955)$ & $(0.085)$ & $(0.085)$ & $(0.051)$ \\
\hline \multicolumn{7}{|c|}{ Simulated records with $(\alpha=1.5$ and $\beta=0.5)$} \\
\hline \multirow[t]{2}{*}{3} & 0.291 & 0.364 & 0.548 & 0.684 & 0.648 & 0.667 \\
\hline & $(0.478)$ & $(0.572)$ & $(0.902)$ & $(0.276)$ & $(0.259)$ & $(0.267)$ \\
\hline \multirow[t]{2}{*}{5} & 0.121 & 0.278 & 0.444 & 0.529 & 0.619 & 0.358 \\
\hline & $(0.235)$ & $(0.409)$ & $(0.115)$ & $(0.148)$ & $(0.206)$ & $(0.122)$ \\
\hline \multirow[t]{2}{*}{7} & 0.012 & 0.099 & 0.852 & 0.532 & 0.457 & 0.175 \\
\hline & $(0.183)$ & $(0.185)$ & (1.655) & $(0.183)$ & $(0.107)$ & $(0.051)$ \\
\hline
\end{tabular}

*The values between brackets is the average mean square error. 
Table 2.

\begin{tabular}{|c|c|c|c|c|c|c|}
\hline \multicolumn{7}{|c|}{ Relative absolute error and mean square error for $\hat{\alpha}$ and $\hat{\beta}$} \\
\hline$m$ & $\hat{\alpha}$ & $\hat{\alpha}_{4}$ & $\hat{\alpha}_{5}$ & $\hat{\beta}$ & $\hat{\beta}_{2}$ & $\hat{\beta}_{3}$ \\
\hline \multicolumn{7}{|c|}{ Simulated records with $(\alpha=0.5$ and $\beta=1)$} \\
\hline \multirow[t]{2}{*}{3} & 0.173 & 0.088 & 0.52 & 0.188 & 0.167 & 0.254 \\
\hline & $(0.195)$ & $(0.154)$ & $(0.306)$ & $(0.188)$ & $(0.174)$ & $(0.214)$ \\
\hline \multirow[t]{2}{*}{5} & 0.464 & 0.153 & 0.827 & 0.127 & 0.163 & 0.162 \\
\hline & $(0.237)$ & $(0.156)$ & $(0.422)$ & $(0.152)$ & $(0.142)$ & $(0.156)$ \\
\hline \multirow[t]{2}{*}{7} & 0.747 & 0.189 & 1.192 & 0.094 & 0.25 & 0.121 \\
\hline & $(0.341)$ & $(0.202)$ & $(0.66)$ & $(0.096)$ & $(0.189)$ & $(0.113)$ \\
\hline \multicolumn{7}{|c|}{ Simulated records with $(\alpha=1$ and $\beta=1)$} \\
\hline \multirow[t]{2}{*}{3} & 0.047 & 0.131 & 0.369 & 0.234 & 0.221 & 0.254 \\
\hline & $(0.224)$ & $(0.257)$ & $(0.985)$ & $(0.2)$ & $(0.214)$ & $(0.214)$ \\
\hline \multirow[t]{2}{*}{5} & 0.134 & 0.014 & 0.601 & 0.212 & 0.226 & 0.162 \\
\hline & $(0.234)$ & $(0.247)$ & $(0.921)$ & $(0.172)$ & $(0.179)$ & $(0.156)$ \\
\hline \multirow[t]{2}{*}{7} & 0.335 & 0.105 & 0.956 & 0.185 & 0.267 & 0.122 \\
\hline & $(0.253)$ & $(0.202)$ & (1.533) & $(0.108)$ & $(0.168)$ & $(0.113)$ \\
\hline \multicolumn{7}{|c|}{ Simulated records with $(\alpha=1.5$ and $\beta=1)$} \\
\hline \multirow[t]{2}{*}{3} & 0.177 & 0.258 & 0.375 & 0.276 & 0.246 & 0.254 \\
\hline & $(0.281)$ & $(0.369)$ & $(1.01)$ & (0209.) & $(0.223)$ & $(0.214)$ \\
\hline \multirow[t]{2}{*}{5} & 0.058 & 0.168 & 0.54 & 0.335 & 0.295 & 0.162 \\
\hline & $(0.149)$ & $(0.223)$ & (1.7) & $(0.225)$ & $(0.2)$ & $(0156)$. \\
\hline \multirow[t]{2}{*}{7} & 0.066 & 0.039 & 0.891 & 0.307 & 0.311 & 0.122 \\
\hline & $(0.091)$ & $(0.127)$ & (1.891) & $(0.176)$ & $(0.165)$ & $(0.113)$ \\
\hline
\end{tabular}

*The values between brackets is the average mean square error. 
Table 3.

\begin{tabular}{|c|c|c|c|c|c|c|}
\hline \multicolumn{7}{|c|}{ Relative absolute error and mean square error for $\hat{\alpha}$ and $\hat{\beta}$} \\
\hline$m$ & $\hat{\alpha}$ & $\hat{\alpha}_{4}$ & $\hat{\alpha}_{5}$ & $\hat{\beta}$ & $\hat{\beta}_{2}$ & $\hat{\beta}_{3}$ \\
\hline \multicolumn{7}{|c|}{ Simulated records with $(\alpha=0.5$ and $\beta=1.5)$} \\
\hline \multirow[t]{2}{*}{3} & 0.541 & 0.308 & 0.931 & 0.065 & 0.049 & 0.027 \\
\hline & $(0.246)$ & $(0.174)$ & $(0.459)$ & $(0.125)$ & $(0.118)$ & $(0.106)$ \\
\hline \multirow[t]{2}{*}{5} & 0.8 & 0.4 & 1.227 & 0.056 & 0.008 & 0.044 \\
\hline & $(0.34)$ & $(0.184)$ & $(0.598)$ & $(0.111)$ & $(0.099)$ & $(0.098)$ \\
\hline \multirow[t]{2}{*}{7} & 0.94 & 0.685 & 1.507 & 0.018 & 0.006 & 0.017 \\
\hline & $(0.406)$ & $(0.289)$ & $(0.849)$ & $(0.086)$ & $(0.085)$ & $(0.093)$ \\
\hline \multicolumn{7}{|c|}{ Simulated records with $(\alpha=1$ and $\beta=1.5)$} \\
\hline \multirow[t]{2}{*}{3} & 0.149 & 0.078 & 0.595 & 0.036 & 0.058 & 0.026 \\
\hline & $(0.195)$ & $(0.186)$ & $(1.113)$ & $(0.106)$ & $(0.127)$ & $(0.106)$ \\
\hline \multirow[t]{2}{*}{5} & 0.33 & 0.214 & 0.845 & 0.007 & 0.011 & 0.044 \\
\hline & $(0.251)$ & $(0.228)$ & $(1.21)$ & $(0.086)$ & $(0.087)$ & $(0.098)$ \\
\hline \multirow[t]{2}{*}{7} & 0.421 & 0.326 & 1.139 & 0.065 & 0.041 & 0.017 \\
\hline & $(0.282)$ & $(0.246)$ & $(1.621)$ & $(0.069)$ & $(0.071)$ & $(0.093)$ \\
\hline \multicolumn{7}{|c|}{ Simulated records with $(\alpha=1.5$ and $\beta=1.5)$} \\
\hline \multirow[t]{2}{*}{3} & 0.062 & 0.137 & 0.481 & 0.003 & 0.047 & 0.026 \\
\hline & $(0.153)$ & $(0.199)$ & $(0.832)$ & $(0.088)$ & $(0.013)$ & $(0.106)$ \\
\hline \multirow[t]{2}{*}{5} & 0.051 & 0.07 & 0.696 & 0.077 & 0.038 & 0.044 \\
\hline & $(0.068)$ & $(0.141)$ & $(0.995)$ & $(0.073)$ & $(0.088)$ & $(0.085)$ \\
\hline \multirow[t]{2}{*}{7} & 0.146 & 0.031 & 0.944 & 0.146 & 0.135 & 0.017 \\
\hline & $(0.095)$ & $(0.063)$ & $(1.32)$ & $(0.083)$ & $(0.103)$ & $(0.093)$ \\
\hline
\end{tabular}

*The values between brackets is the average mean square error.

\section{Conclusion}

Based on the set of upper record values, this paper devoted the Bayesian and non-Bayesian approaches to estimate the two unknown parameters of the Weibull-uniform distribution. The Bayes estimators are obtained using both squared error loss and zero one loss function 
based on upper record values. Comparison was made between the average relative absolute error for the different estimators based on a simulation study using upper record values. Also the average mean square error was examined.

\section{References}

[1] S. Gulati and W. J. Padgett, Smooth nonparametric estimation of the distribution and density function from record breaking data, Commun Statist. Theor. Meth. 23(5) (1994), 1247-1259.

[2] S. I. Resnick, Extreme Values, Regular Variation, and Point Processes, Springer, New York, 1987.

[3] H. N. Nagaraja, Record values and related statistics - A review, Commun. Statist. Theor. Meth. 17 (1988), 2223-2238.

[4] M. Ahsanullah, On the record values from univariate distributions, National Institute of Standards and Technology, J. Res. Special Publications 866 (1993), 1-6.

[5] M. Ahsanullah, Record Statistics, Nova Science Publishers Inc., Huntington, New York, 1995.

[6] B. C. Arnold, N. Balakrishnan and H. N. Nagaraja, A First Course in Order Statistics, Wiley, New York, 1992.

[7] B. C. Arnold, N. Balakrishnan and H. N. Nagaraja, Records, Wiley, New York, 1998.

[8] M. Z. Raqab and M. Ahsanullah, Estimation of the location and scale parameters of generalized exponential distribution based on order statistics, J. Statist. Comput. Simulation 69(2) (2001) 109-124.

[9] A. Soliman, E. A. Amin and A. A. Abdel Aziz, Estimation and prediction from inverse Rayleigh distribution based on lower record values, App. Math. Sci. 4(62) (2010), 3057-3066.

[10] K. N. Chandler, The distribution and frequency of record values, J. Roy. Stat. Soci. Ser. B 14 (1952), 220-228.

[11] W. Feller, An Introduction to Probability Theory and its Applications, Vol. 2, John Wiley and Sons, New York, 1966.

[12] S. I. Resnick, Record values and maxima, Ann. Probab. (1973), 650-662.

[13] N. Balakrishnan and M. Ahsanullah, Recurrence relations for single and product moments of record values from generalized Pareto distribution, Commun. Statist. Theor. Meth. 23(10) (1994), 2841-2852.

[14] M. Bourguignon, R. B. Silva and G. M. Cordeiro, The Weibull-G family of probability distributions, Journal of Data Science 12 (2014), 53-68. 
[15] J. F. Lawless, Statistical Models and Method of Lifetime Data, John Wiley, New York, 1982.

[16] S. K. Ashour and E. A. Amin, Bayesian and non-Bayesian estimation about Weibull distribution based on upper record values, The Egyptian Statistical Journal 49 (2005), 2.

[17] P. Congdon, Bayesian Statistical Modelling, Wiley, New York, 2001. 